\title{
On Laplacian Energy of Certain Mesh Derived Networks
}

\author{
Bharati Rajan \\ Department of Mathematics \\ Loyola College \\ Chennai \\ Albert William \\ Department of Mathematics \\ Loyola College \\ Chennai
}

\author{
Sudeep Stephen \\ Department of Mathematics \\ Loyola College \\ Chennai \\ Cyriac Grigorious \\ Department of Mathematics \\ Loyola College \\ Chennai
}

\begin{abstract}
Eigenvalues of a graph are the eigenvalues of its adjacency matrix. The multiset of eigenvalues is called its spectrum. There are many properties which can be explained using the spectrum like energy, connectedness, vertex connectivity, chromatic number, perfect matching etc. Laplacian spectrum is the multiset of eigenvalues of Laplacian matrix. The Laplacian energy of a graph is the sum of the absolute values of its Laplacian eigenvalues. In this paper we calculate the Laplacian energy of some grid based networks
\end{abstract}

\section{Keywords}

Grid, cylinder, torus, energy, Laplacian energy

\section{INTRODUCTION}

Spectral methods in graph theory have received great attention since their introduction and have proved to be a valuable tool for the theoretical and applied graph theory [1,2]. The study of graph eigenvalues realizes increasingly rich connections with many other areas of mathematics.

A graph $G$ with vertex set $V=\left\{v_{1}, v_{2} \ldots v_{n}\right\}$ can be represented by its adjacency matrix $A=A(G)=\left[a_{i j}\right]$ where $a_{i j}=1$ if $v_{i}$ and $v_{j}$ are adjacent and $a_{i j}=0$ otherwise. The spectrum of $G$ is the set of numbers which are eigenvalues of $A(G)$, together with their multiplicities. If the distinct eigenvalues of $A(G)$ are $\lambda_{0}>\lambda_{1}>\ldots>\lambda_{s-1}$ and their multiplicities are $m\left(\lambda_{0}\right), m\left(\lambda_{1}\right), \ldots, m\left(\lambda_{s-1}\right)$, then we can write the spectrum as

$$
\operatorname{Spec} G=\left(\begin{array}{ccccc}
\lambda_{0} & \lambda_{1} & \cdot & \cdot & \lambda_{s} \\
m\left(\lambda_{0}\right) & m\left(\lambda_{1}\right) & \cdot & \cdot & m\left(\lambda_{s-1}\right)
\end{array}\right)
$$

The energy $E(G)$ of a simple graph $G$ is defined to be the sum of the absolute values of the eigenvalues of $G$. In [3] the spectra and energy of several classes of graphs containing a linear polyene fragment are obtained. In [4] the energy of iterated line graphs of regular graphs and in [5] the energy of some self complimentary graphs are discussed. The energy of regular graphs is discussed in [6]. Some works pertaining to the computation of energy can be seen in $[7,8,9,10,11]$.

The Laplacian matrix $L=D-A$ is the difference of $D=\operatorname{diag}\left(d_{1}, d_{2}, \ldots, d_{n}\right)$, the diagonal matrix of vertex degrees, and the adjacency matrix $A$. The matrix $L$ is positive semi-definite, and therefore its eigenvalues are non-negative. The least eigenvalue is always equal to 0 ; the second least eigenvalue is also called the algebraic connectivity of $G$ [12]. Let $\mu_{1} \geq \mu_{2} \geq \cdots \geq \mu_{n} \geq 0$ be the Laplacian eigenvalues of $G$.
The Laplacian energy, denoted by $L E(G)$, is defined as $L E(G)=\sum_{i=1}^{n}\left|\mu_{i}-\frac{2 m}{n}\right|$

It has been found [13] that there are a number of analogous properties between $E(G)$ and $L E(G)$. It is known that if the graph $G$ is regular, then $L E(G)=E(G)$.

\section{APPLICATIONS}

Motivation for founding the theory of graph spectra has come from applications in Chemistry and Physics. The first mathematical paper on graph spectra was motivated by the membrane vibration problem [14]. In quantum chemistry, the skeleton of a non-saturated hydrocarbon is represented by a graph. The energy levels of the electrons in such a molecule are the eigenvalues of the graph. The stability of molecules is closely related to the spectrum of its graph [15]. The spectra of graphs, or the spectra of certain matrices which are closely related to adjacency matrices appear in a number of problems in statistical physics (see, for example, $[16,17,18]$ ). Graph spectra appear in internet technologies, pattern recognition, computer vision, and in many other areas. In geographic studies, the eigenvalues and eigenvectors of a transportation network provide information about its connectedness. It is proved that the more highly connected the transportation network $G$ is, the larger is the, the largest eigenvalue $\lambda_{1}(G)$. $[19,20]$

The eigenvalues of a graph characterize the topological structure of the graph. The technique is usually efficient in counting structures, e.g., acyclic digraphs, spanning trees, Hamiltonian cycles, independent sets, Eulerian orientations, cycle covers, $k$-colorings etc. If a graph possesses certain property, using its eigen-properties it is possible to derive (recurrence) formulas for counting the number of structures.

As there is no easy way to determine the eigenvalues of a graph $\mathrm{G}$, determining the complete spectrum is an interesting problem. Here in this paper we obtain the spectrum of interconnection networks such as grid $G(n, n)$, cylinder $C(n, n)$, torus $T R(n, n)$ using MATLAB.

\section{CALCULATING THE ENERGY}

\subsection{Grid $G(l, n)$}

The topological structure of a grid network, denoted by $G(l, n)$, is defined as the Cartesian product $P_{l} \times P_{n}$ of undirected paths $P_{l}$ and $P_{n}$. For convenience we assume that $l=n$. The spectrum of the graph does not depend on the numbering of the vertices. However here we adopt a particular numbering such that the adjacency matrix has a pattern which is common for any dimension. We follow the sequential 
numbering from left to right. The following diagram illustrates the numbering chosen.

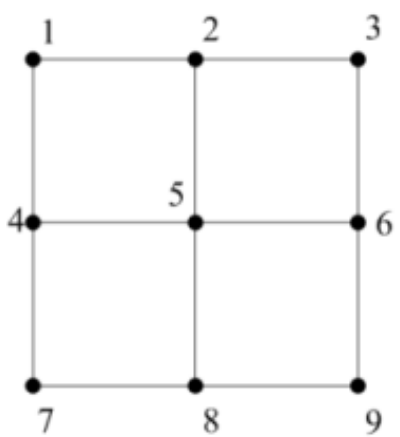

Figure 1: Sequential numbering of Grid $(3,3)$

The adjacency matrix of $G(3,3)$ is given by

$A=\left(\begin{array}{lll}B & I & O \\ I & B & I \\ O & I & B\end{array}\right)$ where $B=\left(\begin{array}{ccc}0 & 1 & 0 \\ 1 & 0 & 1 \\ 0 & 1 & 0\end{array}\right), I$ is the

identity matrix of size $3 \times 3$ and $O$ is the zero matrix of size $3 \times 3$.

In general the adjacency matrix of $G(n, n)$ can be written as

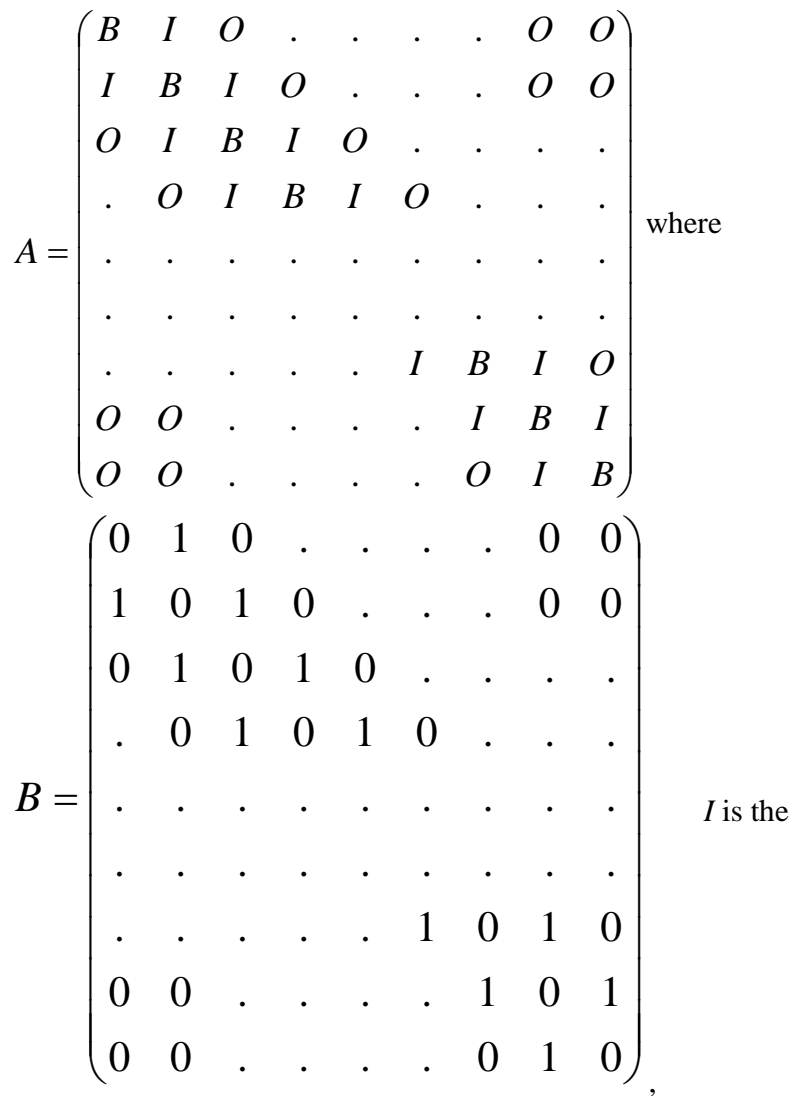

identity matrix and $O$ is the zero matrix of size $n \times n$.

The following MATLAB program generates the adjacency matrix of grid $G(n, n)$ and also calculates its energy.

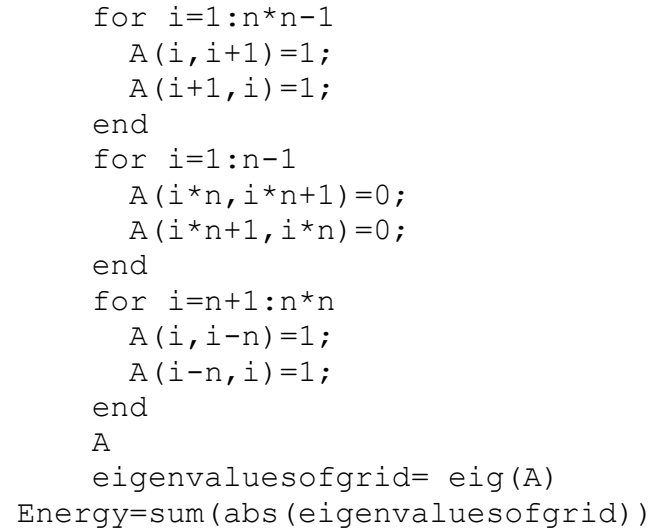

\section{Proof of correctness of energygrid program}

It can be easily seen from the MATLAB program that the first and the second 'for loop' generate the matrices $B$ 's along the main diagonal in the adjacency matrix. The $(i, i)^{\text {th }}$ entry $B$ of the block representation of the adjacency matrix corresponds to the $i^{\text {th }}$ row of the grid which is a path on $n$ vertices. The last 'for loop' generates the identity matrices I's along both sides of the main diagonal in the block representation and accounts for the column edges of the grid between the paths.

In order to calculate the Laplacian matrix, we devise a logic to write down the degree matrix $D$. First we consider a diagonal matrix $D=4 I$, where $I$ is identity matrix of size $n^{2} \times n^{2}$. Replace $\quad D(1,1), D(n, n), D\left(n^{2}-n+1, n^{2}-n+1\right) \quad$ and $D\left(n^{2}, n^{2}\right)$ by 2 . To obtain the degree matrix, we need to replace $4(n-2)$ entries of $\mathrm{D}$ which correspond to the first and last rows and first and last columns of the grid except for the four corner vertices with 3 . The resultant $D$ will be the degree matrix of grid $G(n, n)$. The following MATLAB codes generate the Laplacian Matrix and Laplacian energy of $G(m, m)$

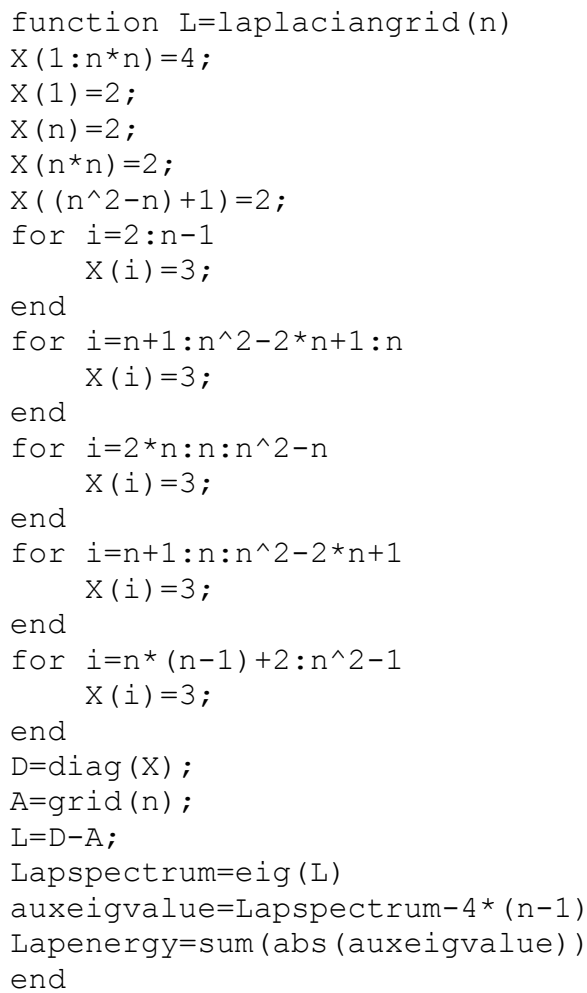




\subsection{Cylinder $C(l, n)$}

The topological structure of a cylinder network, denoted $C(l, n)$ is defined as the Cartesian product $P_{l} \times C_{n}$ of undirected path $P_{l}$ and an undirected cycle $C_{n}$. For convenience we assume that $l=n$. The numbering adopted for cylinder is same as that of grid. The adjacency matrix of $C(n, n)$ in block form is

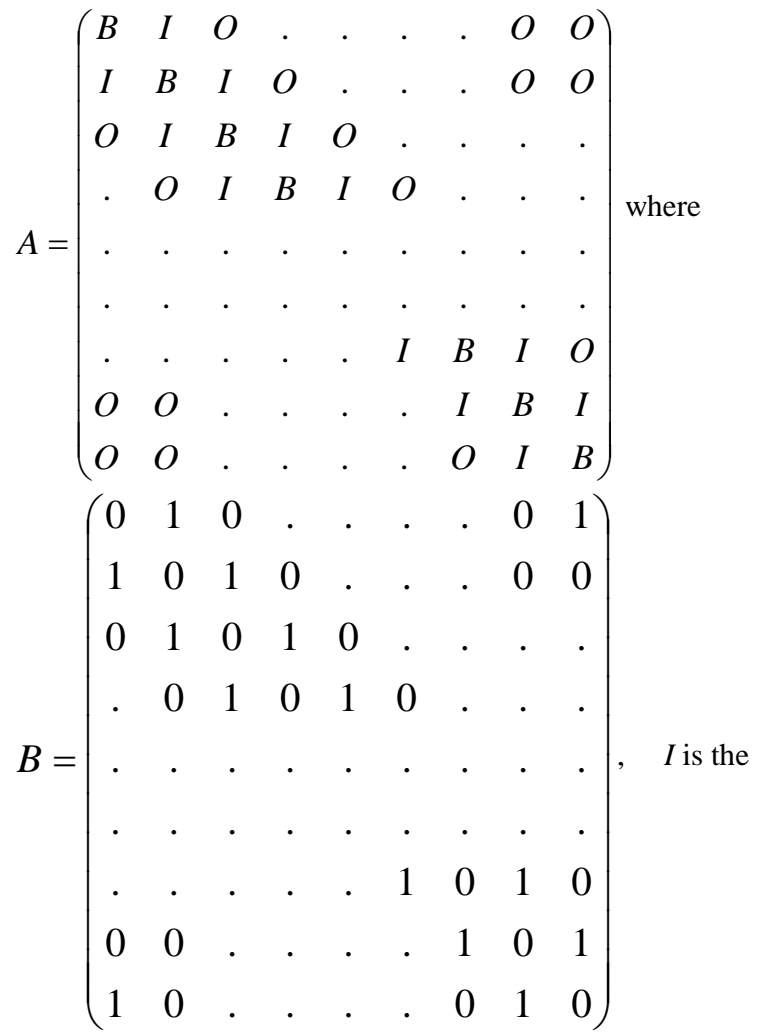

identity matrix of size $n \times n$ and $O$ is the zero matrix of size $n \times n$. The following MATLAB program generates the adjacency matrix of cylinder $C(n, n)$ and calculates its energy.

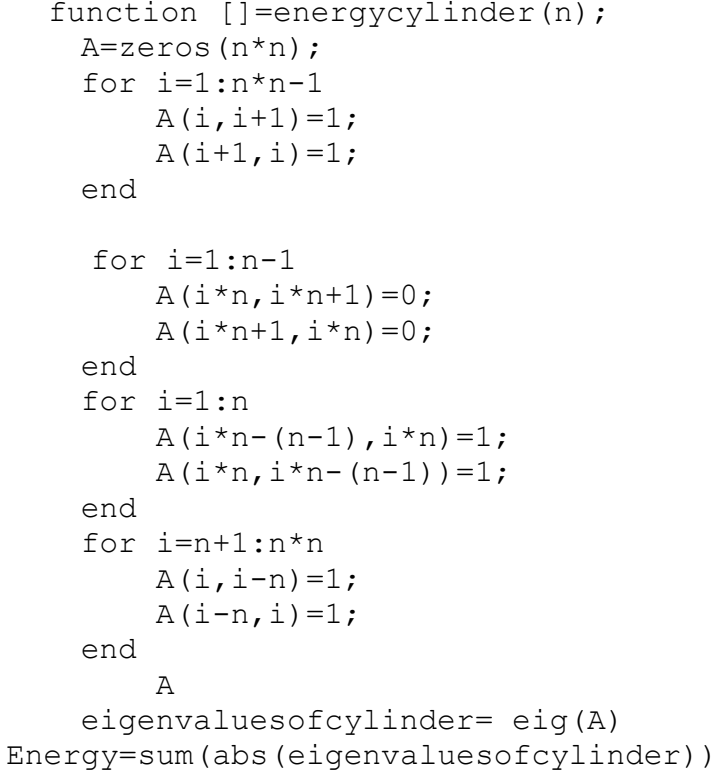

Proof of correctness of the above programme is similar to the first one, that we skip for the readers to prove. Obtaining the Laplacian matrix and hence Laplacian energy becomes easier after generating the adjacency matrix. The following
MATLAB function calculates the Laplacian energy of $C(n, n)$.

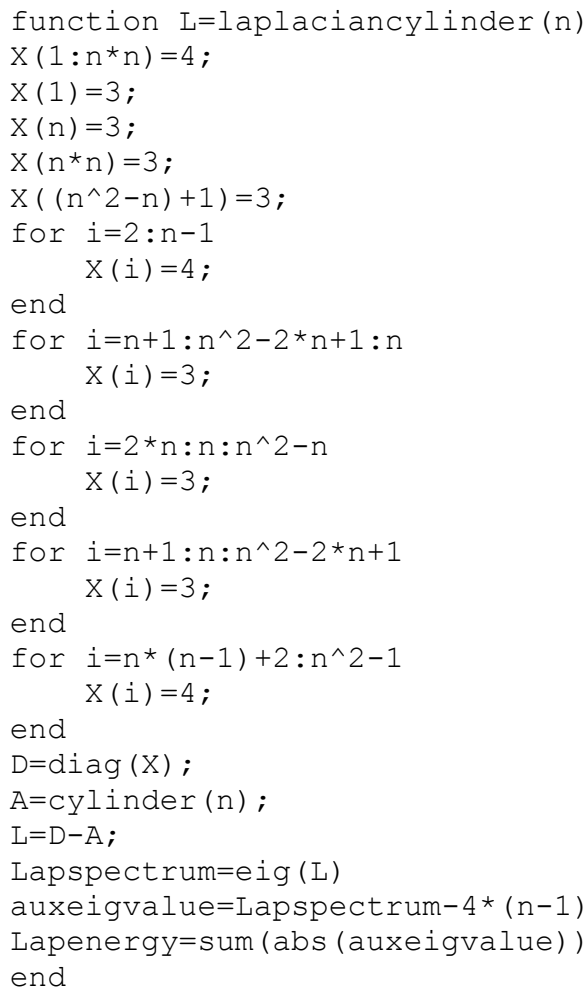

\subsection{Torus $T R(l, n)$}

The topological structure of a torus network, denoted $T R(l, n)$ is defined as the Cartesian product $C_{l} \times C_{n}$ where $C_{l}$ and $C_{n}$ are undirected cycles. The numbering adopted for torus is same as that of grid i.e., sequential labelling from left to right The adjacency matrix of $T R(n, n)$ in block form is

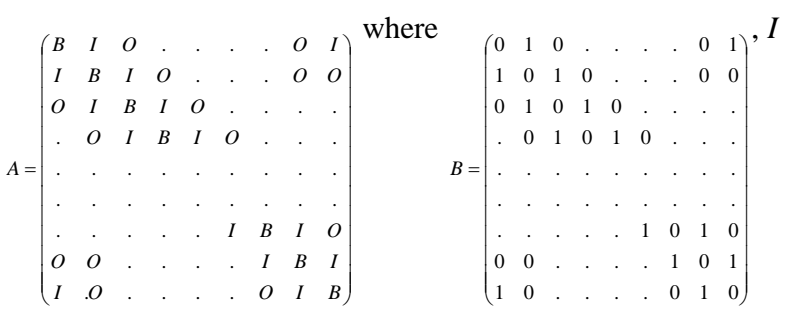

is the identity matrix and $O$ is the zero matrix of size $n \times n$. The following MATLAB program generates the adjacency matrix of torus $T R(n, n)$ and also calculates its energy.

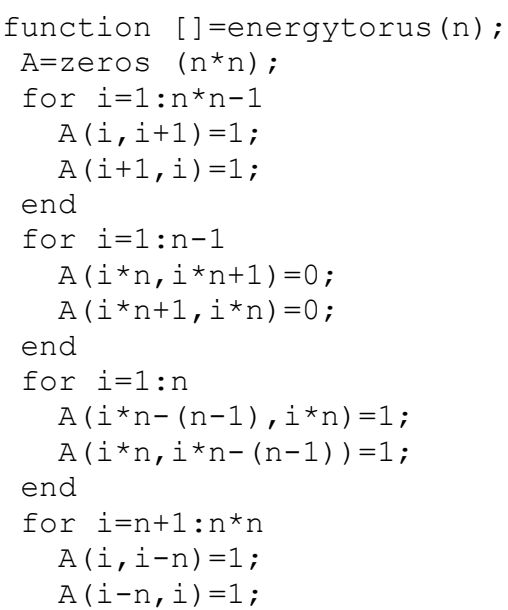




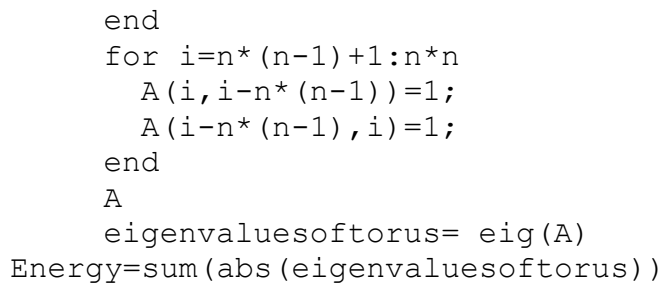

The following MATLAB commands gives the Laplacian Matrix and Laplacian energy of $T R(n, n)$.

function L=laplaciantorus ( $\mathrm{n}$ )

$\mathrm{X}(1: \mathrm{n} * \mathrm{n})=4$

$\mathrm{D}=\operatorname{diag}(\mathrm{X})$;

$\mathrm{A}=\operatorname{torus}(\mathrm{n})$

$\mathrm{L}=\mathrm{D}-\mathrm{A}$;

Lapspectrum=eig $(\mathrm{L})$

auxeigvalue=Lapspectrum $4 * n$

Lapenergy=sum (abs (auxeigvalue))

End

\subsection{Extended grid $E X(l, n)$}

By making each 4-cycle in a $l \times n$ mesh into a complete graph we obtain an architecture called an extended mesh denoted by $E X(l, n)$. The number of vertices in $E X(l, n)$ is $l n$ and the number of edges in $E X(l, n)$ is $4 l n-3 l-3 n+2$. We follow the sequential numbering from left to right.

The following diagram illustrates the numbering chosen.

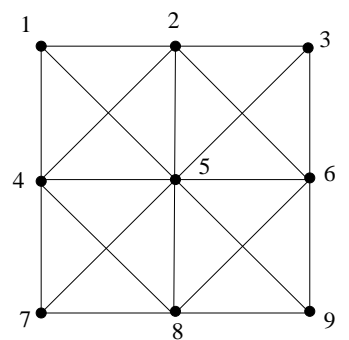

Figure 2: Sequential labelling of $E X(3,3)$

The adjacency matrix of $E X(3,3)$ is given

$$
\text { by } A=\left(\begin{array}{lll}
B & C & O \\
C & B & C \\
O & C & B
\end{array}\right)^{\text {? }}
$$

where

$$
B=\left(\begin{array}{lll}
0 & 1 & 0 \\
1 & 0 & 1 \\
0 & 1 & 0
\end{array}\right)_{\text {回, }} \quad C=\left(\begin{array}{lll}
1 & 1 & 0 \\
1 & 1 & 1 \\
0 & 1 & 1
\end{array}\right)
$$

and $O$ is the zero matrix of size $3 \times 3$.

In general the adjacency matrix of $E X(n, n)$ can be written as

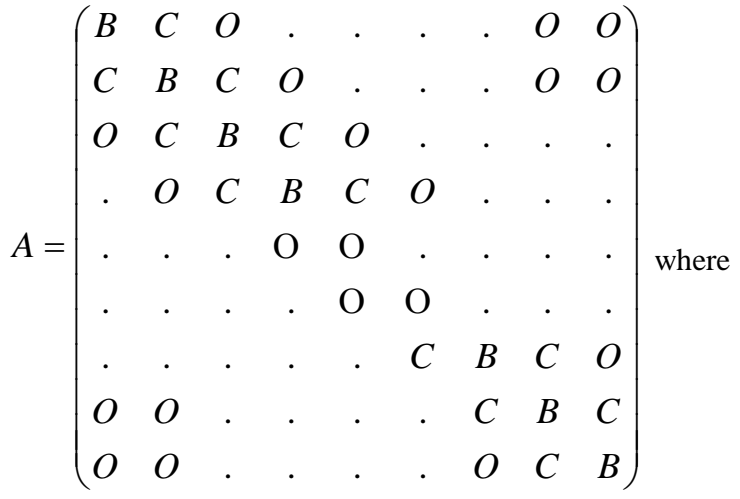

$B=\left(\begin{array}{lllllllll}0 & 1 & 0 & . & . & . & . & 0 & 0 \\ 1 & 0 & 1 & 0 & . & . & . & 0 & 0 \\ 0 & 1 & 0 & 1 & 0 & . & . & . & . \\ . & 0 & 1 & 0 & 1 & 0 & . & . & . \\ . & . & . & . & . & . & . & . & . \\ . & . & . & . & . & . & . & . & . \\ . & . & . & . & . & 1 & 0 & 1 & 0 \\ 0 & 0 & . & . & . & . & 1 & 0 & 1 \\ 0 & 0 & . & . & . & . & 0 & 1 & 0\end{array}\right)$,

$C=\left(\begin{array}{lllllllll}1 & 1 & 0 & . & . & . & . & 0 & 0 \\ 1 & 1 & 1 & 0 & . & . & . & 0 & 0 \\ 0 & 1 & 1 & 1 & 0 & . & . & . & . \\ . & 0 & 1 & 1 & 1 & 0 & . & . & . \\ . & . & . & . & . & . & . & . & . \\ . & . & . & . & . & . & . & . & . \\ . & . & . & . & . & 1 & 1 & 1 & 0 \\ 0 & 0 & . & . & . & . & 1 & 1 & 1 \\ 0 & 0 & . & . & . & . & 0 & 1 & 1\end{array}\right)$

and $O$ is the zero matrix of size $n \times n$. The following MATLAB program generates the adjacency matrix of extended mesh $E X(n, n)$ and also calculates its energy.

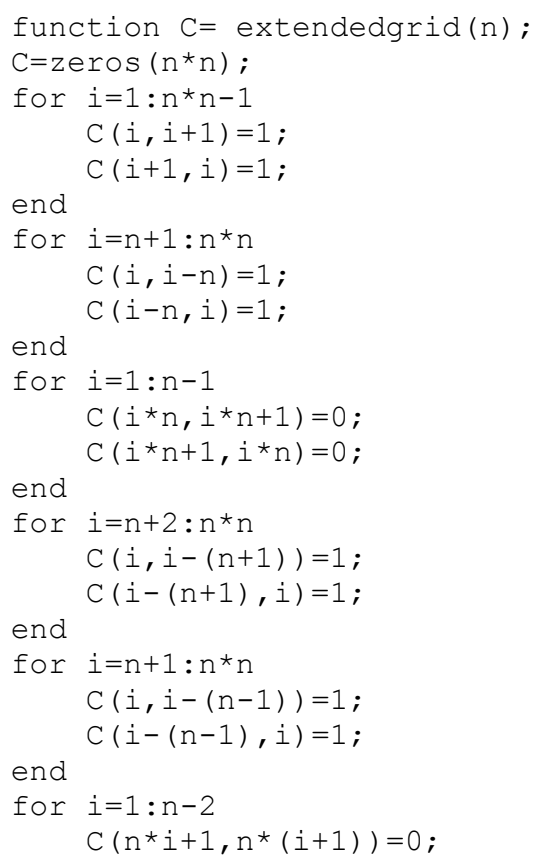




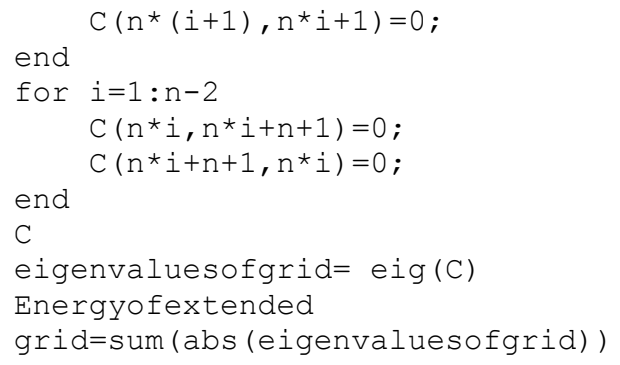

The following MATLAB commands give the Laplacian Matrix and Laplacian energy of $E X(n, n)$.



\section{ACKNOWLEDGEMENT}

This research is supported by Maulana Azad Fellowship F117.1/2010/MANF-CHR-KER-4749 of the University Grants Commission, New Delhi, India.

\section{CONCLUSION}

In this paper we have devised a method to compute the spectrum and thereby the energy and Laplacian energy of mesh derived networks of all dimensions. This is of great relevance in studying the structural properties of these networks in a new perspective. Finding the energy of certain interconnection networks such as butterfly, benes network and other interconnection networks are under investigation.

\section{REFERENCES}

[1]. Biggs, N. (1993). L. Algebraic Graph Theory. Cambridge University Press, Cambridge. 2nd ed.,.
[2]. Chung, F.R. (1994). Spectral Graph Theory. American Mathematical Society.

[3]. Gutman, I. (1976). A graph theoretical study of conjugated systems containing a linear polyene fragments. Croat. Chem.Acta.48(2), 97-108,

[4]. Ramane, Walikar, Rao, Acharaya, Gutman, Hampiholi, (2004). Equienergetic graph. Kragujevac. J. Math.,26, 513.

[5]. Indulal, G., Vijayakumar (2002). Equienergetic selfcomplimentary graphs. Czechoslovak Math. J,

[6]. Gutman, I., Zare Firoozabadi, J.A. de la Peňa, Rada (2007). On the energy of regular graphs. MATCH Commun. Math. Comput. Chem., 57, 435-442.

[7]. Balakrishnan, R (2004). The energy of a graph. Linear Algebra Appl. 387, 287-295.

[8]. Gutman, I. (1978). The energy of a graph. Ber. Math. Stat. Sekt. Forschungszenturm Graz. 103, 1- 22.

[9]. Gutman, I., Milun, Trinajstić (1972). Hůckel Molecular Orbital Calculations of Aromatic Stabilization of Annulenes. Croat. Chem. Acta. 44, 207-213.

[10].Indulal,G., Vijayakumar (2006). On a pair of equienergetuc graphs. MATCH Commun. Math. Comput. Chem.. 55, 83- 90.

[11].Indulal, G., Vijayakumar (2008). A note on energy of some graphs. MATCH Commun. Math. Comput. Chem., 59, 269- 274.

[12].M. Fiedler (1973). Algebraic connectivity of graphs, Czech. Math. J., 23 (98), 298-305.

[13]. Gutman I, Zhou B. (2006). Laplacian energy of a graph, Linear Algebra and its Applications, 414, 29-37,.

[14].Collatz L., Sinogowitz U. (1957). Spektren endlicher Grafen. Abh. Math. Sem. Univ. Hamburg. 21, 63-77,

[15].Cvetkovi'c, D. M., Doob, M., Sachs, H. (1995) Spectra of graphs. Johann Ambrosius Barth Verlag, Third edition.

[16].Kasteleyn (1967). Graph theory and crystal psysics. Graph theory and theoretical physics (ed. F. Harary), London, 43-110,

[17].Montroll, E. W. (1964). Lattice statistics. Applied combinatorial mathematics. Wiley, New York,(E. F. Becken-bach, Ed.), 96-143.

[18].Percus, J. K. (1969). Combinational Methods. SpringerVerlag. Berlin-Heidelberg-New York.

[19].Tinkler, K. (1972). The physical interpretation of eigenvalues of dichotomous matrix. Inst. Brit. Geogr Puli., 55, 17-46.

[20].Roberts, F. (1978). Graph Theory and Its Applications to Problems of Society. Soc. Ind. App.Math.,Philadelphia. 